\section{Inborn errors of metabolism}

J. Arnemann

Abteilung Molekulargenetik, Labor Dr. Wisplinghoff, Köln, Deutschland

Synonym(e) angeborene; Stoffwechselerkrankungen

Englischer Begriff inborn errors of metabolism

Definition Unter dem Begriff, ,inborn errors of metabolism“ verbirgt sich analog zur Syndromdiagnostik das große Gebiet der angeborenen Stoffwechselerkrankungen.

Beschreibung Angeborene Stoffwechselstörungen fallen meist bereits frühkindlich klinisch auf durch eine Akkumulation toxischer Stoffwechselprodukte oder Reduktion essenzieller Stoffwechselkomponenten aufgrund eines Enzymdefekts. Man schätzt, dass zu dieser Gruppe ca. $15 \%$ aller Einzelgendefekte gehören. Die klinische Diagnose wird meist durch biochemische Analysen und/oder molekulargenetische Untersuchungen der Kandidatengene bestätigt. Der Nachweis der molekularen Basisdefekte hilft bei der Familienplanung und einer gegebenenfalls gezielten Pränataldiagnostik. Einige der Erkrankungen sind mittlerweile auch Bestandteile des Neugeborenenscreenings ( $\triangleright$ Neugeborenenscreening auf Stoffwechselerkrankungen und Endokrinopathien), insbesondere auch aufgrund der technischen Weiterentwicklung, wie z. B. GC-MS ( $>$ Gaschromatographie-Massenspektrometrie).

Die Gruppe der angeborenen Stoffwechselstörungen ist nach den betroffenen metabolischen Stoffgruppen unterteilt, wie z. B.
- Störungen des Kohlenhydratstoffwechsels (z. B. Glykogenspeicherkrankheiten)

- Störungen des Aminosäurestoffwechsels (z. B. Phenylketonurie)

- Störungen des Harnstoffzyklus (z. B. CarbamoylphosphatSynthetase Typ I)

- Störungen im Stoffwechsel organischer Säuren (z. B. Alkaptonurie)

- Störungen der Fettsäureoxidation und des mitochondrialen Stoffwechsels (z. B. MCAD-Defizienz)

- Störungen des Porphyrinstoffwechsels (z. B. akute intermittierende Porphyrie)

- Störungen im Purin- und Pyrimidinstoffwechsel (z. B. Lesh-Nyan-Syndrom)

- Störungen des Steroidstoffwechsels (z. B. kongenitale adrenale Hyperplasie)

- Störungen der Mitochondrienfunktion (z. B. KearnsSayre-Syndrom)

- Störungen in der Peroxisomenfunktion (z. B. ZellwegerSyndrom)

- Störungen als Lysosomspeicherkrankheiten (z. B. Morbus Gaucher)

\section{Literatur}

Lanpher et al (2006) Inborn errors of metabolism: the flux from Mendelian to complex diseases. Nat Rev Genet 7:449-459

Wilcken et al (2003) Screening newborns for inborn errors of metabolism by tandem mass spectrometry. NEJM 348:2304-2312 